\title{
Zu satzfinaler Subjektposition, Unakkusativität und C-Domäne im Jiddischen*
}

\author{
Pawel Mecner (Stettin)
}

\begin{abstract}
The article examines structures of Yiddish clauses in which the kernel verb contains only one NP. The thematic role of an accessible NP is patient/theme, whereas the agent-NP remains vacant. It is assumed that a direct object of the type "X efent $Y$ " ('X opens $Y$ ') and an accessible NP of the type "exists Y" appear in Yiddish in the clause-final position as a focalized subject. The study combines minimalist investigations and the cartographic approach, especially with regard to CP and IP/TP structures proposed in Rizzi (1997, 2004) and Belletti (1999) among others, and adopts the concept of functional topic/focus projections in the syntax of Yiddish. Investigations reveal possibilities of case assignment and feature checking in lower projection areas as well as the correlation between the topic projection of the C-domain and the clause-internal focus projection. A mechanism is assumed to combine precisely clause-final focalized subjects, unaccusatives and the $\mathrm{C}$-domain including the following alternatives: (i) unmarked adverbials (PPs), (ii) expletive es and (iii) verb fronting. The analyzed structures have been observed in texts of Isaac Bashevis Singer (1931), Isaac Leib Peretz, (ed. 1920) and Fishl Bimko (1921) as well as in Yiddish daily newspaper Forverts.
\end{abstract}

\section{$1 \quad$ Einleitung}

Jiddisch ist als Untersuchungsgegenstand besonders anregend, da es einerseits der Typologie der germanischen Sprachen angehört und andererseits Einflüsse anderer Sprachfamilien ${ }^{1}$ enthält. Der syntaktische Status von Jiddisch ist allerdings umstritten, weil komplexere Daten aus jiddischen Korpora nur in einem geringeren Grad für syntaktische Analysen ${ }^{2}$ herangezogen wurden. Eine Wende in dieser Hinsicht bringen in dem letzten Jahrzehnt ein komplexer Überblick über die jiddische Sprache von Jacobs (2005) und Ansätze zur Syntax

\footnotetext{
* Für Überarbeitungsvorschläge, die mir geholfen haben, den ursprünglichen Text wesentlich zu ändern, bin ich drei anonymen Gutachtern zu Dank verpflichtet wie auch Elke Hentschel, die dies organisatorisch leitete. Ich danke Marion Aptroot für Kommentare und Verbesserungsvorschläge bezüglich der Fragmente, die sich auf die Darstellung und Übersetzung des jiddischen Materials beziehen. Für Kommentare und Verbesserungsvorschläge danke ich auch Gisbert Fanselow, Anna Pilarski und Magdalena Feret. Alle Fehler sind selbstverständlich die meinigen.

${ }^{1}$ Cf. den Überblick über die historische Entwicklung der jiddischen Sprache und die Diskussion dazu in Beider (2013).

${ }^{2} \mathrm{Cf}$. auch die englischsprachige Literatur in weiteren Abschnitten der Einleitung.
} Linguistik online 80, 1/17 - http://dx.doi.org/10.13092/lo.80.3566

CC by 3.0 
des Jiddischen in Aptroot und Hansen (2014). Fragen zu syntaktischen Mechanismen im Jiddischen bleiben allerdings weiterhin offen.

Ziel des vorliegenden Aufsatzes ist es, Aspekte einer im Korpus des Jiddischen gefundenen syntaktischen Strukturierung zu analysieren. Es kann beobachtet werden, dass das Subjekt im Jiddischen auch am Satzende positioniert werden kann. Dies scheint aber bei unakkusativischen Verben häufiger $\mathrm{zu}$ sein als bei anderen Verbgruppen. Zugleich wird beobachtet, dass diese Art von Subjekt kein Agens ist, sondern die thematische Rolle Patiens/Thema trägt, wie dies bei Objekten transitiver Verben der Fall ist. Die Positionierung von Subjekt beeinflusst auch die Informationsstruktur eines Satzes. Steht das Subjekt am Satzende, ist es fokussiert. In dieser Struktur wird eine präverbale PP zu einem unmarkierten Topik. Alternativ kann vor dem Finitum das Expletiv es stehen oder das Finitum kann zu deklarativem V1 werden. Dies scheint ein eigenständiger syntaktischer Mechanismus zu sein, der satzfinale Subjektpositionen, unakkusativische Verben und C-Domäne verzahnt, und der sich von einer pragmatisch geregelten Topik/Fokus-Distribution und Akzentsetzung unterscheidet.

Für eine mögliche Erklärung dieses Phänomens im Jiddischen ist die Einbeziehung von Topik/Fokus in das syntaktische Modell von Vorteil. Rizzi (1997, 2004) schlägt eine neue Strukturierung der C-Domäne vor (cf. auch Kapitel 2 im folgenden Text), in der Topik und Fokus als Projektionen funktionaler Köpfe spezifiziert werden, cf. ...Force... (Topik) ... (Focus) ... Fin (IP), (Rizzi 1997: 288). Belletti (1999, 2004) kopiert das Topik/Fokus-System der linken Satzperipherie in den tieferen IP-Bereich. Für das analysierte Phänomen des Jiddischen kann dieses Konzept nach Modifikationen übernommen werden.

Das satzfinale Subjekt, das die thematische Rolle Patiens/Thema trägt, kann auch auf die Diskussion bezogen werden, ob Jiddisch eine SOV- oder SVO-Sprache ist. In der einschlägigen Literatur zur Syntax werden Argumente sowohl für die SVO-Abfolge im Jiddischen (Diesing 1990, 1997, 2005) als auch für SOV (u. a. Geilfuß 1991; Sadock 1998, Wallenberg 2013; Vikner 2013) gebracht. Wallenberg (2013: 290) nimmt in Anlehnung an diachronische Untersuchungen von Santorini $(1992,1993)$ an, dass sich Jiddisch von einer kopffinalen zu einer TP-medialen Sprache in der Periode 1400-1800 entwickelte. Während dieses Prozesses überlappten sich beide Varianten (cf. auch die Analyse von Belegen in Kühnert/Wagner 2014). Trotz dieser Wandlung nimmt Wallenberg (2013: 291) an, dass Jiddisch in der Struktur $v$ P weiterhin OV ist. Haider und Szucsich (2012) schlagen eine dritte Lösung mit einem mobilen V für slawische Sprachen vor, die eine Diskussion auch für das Jiddische eröffnet. Da das satzfinale Subjekt bei unakkusativischen Verben im Jiddischen als ein im syntaktischen Mechanismus umstrukturiertes Objekt transitiver Verben betrachtet werden kann, wird diesbezüglich eine zugrundeliegende VO-Basisstruktur angenommen. Diese Strukturierung kann auch als ein Argument für die VO-Abfolge im Jiddischen angesehen werden.

Im Folgenden werden Konstruktionen mit unakkusativischen Verben und satzfinalem Subjekt analysiert, deren C-Domäne adverbiale PPs, Expletiv es oder Verb(komplex) V1 enthält: (i) 
$P P(A d v)^{3} V_{\text {fin }}$ Subjekt, (ii) Expletiv $V_{\text {fin }}$ Subjekt, (iii) $V_{\text {fin }}$ Subjekt. Die empirische Basis umfasst authentische Texte aus der Tageszeitung Forverts ${ }^{4}$ wie auch literarische Texte von Isaac Bashevis Singer (1931), Isaac Leib Peretz (1920) und Fishl Bimko (1921)5. Die Originalschrift mit hebräischen Zeichen (von rechts nach links gelesen) wird parallel mit der YIVO-Transliteration angegeben. Das Sprachmaterial ist so gewählt worden, dass angegebene Beispielsätze keinen größeren Kontext brauchen und in der angegebenen Form interpretiert werden können.

Der Aufsatz enthält folgende Kapitel: (2) Theoretische Grundlage, in der die Idee von Verknüpfung minimalistischer und kartographischer Annahmen dargestellt wird; (3) Problemstellung, die beobachtete Strukturierung des Jiddischen mit unakkusativischen Verben, satzfinaler Subjektposition und unmarkierter Topik enthält wie auch Fragen nach der Spezifik des angenommenen Mechanismus; (4) Jiddisch als V2/V1-Sprache, satzinitial stehende Elemente und Informationsstruktur; (5) Satzfinale Subjektposition als Phänomen des Jiddischen mit folgenden Varianten: (5.1) mit einer PP in der C-Domäne und unakkusativischen Verben, bei welchen zikh (i) als formaler Marker einer nicht-kausativen Lesart genutzt wird und (ii) bei welchen zikh auf einen unbestimmten Experiencer [Hum, Indef] verweist; (5.2) mit einer PP in der C-Domäne und unakkusativischen Verben, die das zayn-Auxiliar selegieren; (5.3) mit dem Expletiv es in der C-Domäne und unakkusativischen Verben beider Subgruppen (zikh/zayn); (5.4) mit dem Verb(komplex) in der C-Domäne (deklaratives V1); (6) Fazit, in dem versucht wird, Analysen zusammenzufassen und die Charakteristik der beobachteten Strukturierung aufzuweisen.

\section{Theoretische Grundlage}

Da im Jiddischen Einflüsse von mehreren Sprachfamilien möglich sind (cf. Beider 2013), kann es von Interesse sein, analysierte Strukturen nicht nur deskriptiv zu erfassen, sondern auch Modelle mental verankerter Mechanismen $\mathrm{zu}$ entwickeln versuchen, die - wie angenommen werden kann - sich in Generationen von Muttersprachlern des Jiddischen als ein homogenes System herauskristallisiert hatten.

In den letzten Jahrzehnten sind minimalistische Ansätze zur generativen Syntax diskutiert worden (cf. Chomsky 1995, 2000, 2008, 2013; cf. auch u. a. Grohmann 2009; Boeckx 2011). Das Minimalistische Programm (MP) entwickelt die Theorie von PF (Phonetic Form) und LF (Logical Form) weiter. Die PF umfasst Postulate, wie phonologische/phonetische Strukturen geformt werden, die dann Artikulationsorgane (bzw. motorische Systeme beim Schreiben) verarbeiten. Die LF umfasst die semantische Interpretation einer syntaktischen Struktur, aber auch Mechanismen, die in der PF nicht direkt beobachtbar sind. Das Minimalistische Programm erforscht elementare Mechanismen in der Syntax und setzt voraus, dass einfache syntaktische Operationen rekursiv verwendet werden. Es werden drei grundlegende

\footnotetext{
${ }^{3}$ Es sind adverbiale Präpositionalphrasen und vorangestellte Adverbialsätze. Objektvoranstellung wird hier nicht berücksichtigt.

${ }^{4}$ Das analysierte Phänomen kann sowohl in aktuellen Texten der Tageszeitung Forverts wie auch in literarischen Texten (Erzählungen) aus Anfang und Mitte des 20. Jahrhundert gefunden werden.

${ }^{5}$ Originaltexte habe ich genutzt dank dem Yiddish Book Center Amherst, ich danke besonders Aaron Lansky, Catherine Madsen und Agnieszka Ilwicka.
} 
Operationen unterschieden: (i) Merge verbindet zwei Elemente $(\alpha, \beta)$ und bildet ein komplexeres syntaktisches Objekt. (ii) Agree überprüft Kongruenz und Kasus. (iii) Move ist komplexer als Merge oder Agree und findet statt, wenn eine einfachere Operation nicht möglich ist (cf. Chomsky 2000: 101).

Die Tendenz zur Reduktion bezieht sich im Minimalistischen Programm auch auf funktionale Kategorien. Chomsky (2000:102) nimmt an, dass $\mathrm{zu}$ den elementaren funktionalen Kategorien die Folgenden gehören: C (Satztyp/Force: deklarativ, interrogativ etc.), $\mathrm{T}$ (Tempus) und $v$ (der Kopf von transitiven Konstruktionen), dementsprechend CTv. Als Derivationsphasen gelten allerdings nur $\mathrm{C}$ und $v$. Bei der Ausarbeitung des Phasenbegriffs betrachtet Chomsky (2008: 143) die Phase C als Kürzel für den Satzbereich, den Rizzi (1997) als linke Peripherie bezeichnet. Die Phase $v$ wird mit der komplexen Argumentstruktur (Transitivität, Kausativität) assoziiert (cf. auch Grohmann 2009).

Parallel zur minimalistischen Reduzierung und zur weitgehenden Abstraktion entwickeln sich in generativen Arbeiten Tendenzen zur detaillierten Erfassung von Satzstrukturen, die als kartographische Ansätze ,cartographic approach` benannt wurden (cf. einen Überblick in Shlonsky 2010). In Anlehnung an Pollock (1989), der die Spaltung von IP als Kongruenz(Agr) und Tempusprojektion (T) separater Köpfe angenommen hat, wird der linke Satzbereich immer detaillierter dargestellt. Rizzi (1997) analysiert die linke Satzperipherie und integriert Topik und Fokus in die Syntax. Er postuliert (2004: 6) folgende Köpfe im CPBereich: (1) Illokution oder Satztyp (Force), (2) Finitheit (Tempus, Modus). Zwischen Force und Fin wird der Fokus platziert, der, wie man annimmt, von Topik-Projektionen umgeben ist (cf. Belletti 2004: 16). Bewegungen von Phrasen in eine entsprechende Position haben Konsequenzen für die semantische Interpretation (LF). Die semantische Interpretation (LF) ist also von einer syntaktischen Konfiguration (PF) abhängig.

Belletti (2004: 17) nimmt weiterhin an, dass CP und IP die gleiche Strukturierung haben. Die linke Satzperipherie (CP) hat ihre Entsprechung in IP/TP, die als Satz-Interne-Peripherie bezeichnet wird. Analog hat die SIP eine Fokus-Projektion, die von Topik-Projektionen umgeben ist. Wie bereits angenommen wurde (cf. Belletti 1999), ist Fokus eine funktionale Kategorie, in deren Spezifizierer-Kopf-Relation der Nominativ zugewiesen und Kongruenzmerkmale überprüft werden können (cf. 5.1).

Trotz scheinbarer Unterschiede zwischen der Einfachheit generativer Mechanismen im Minimalistischen Program und der Komplexität kartographischer Repräsentationen sind die beiden Richtungen nicht widersprüchlich (cf. Cinque/Rizzi 2008: 49). Das früher genannte $\mathrm{CT} v$-System von funktionalen Kategorien kann im MP als ein Kürzel für detaillierte Zonen betrachtet werden, das kartographische Studien umspannt.

Im vorliegenden Aufsatz wird versucht, die minimalistische und die kartographische Sichtweise zu verknüpfen und sie auf die Syntax des Jiddischen zu beziehen. Für die Untersuchung analysierter Strukturen des Jiddischen wird das folgende Modell angenommen: 
$(1 \mathrm{a})^{6}$

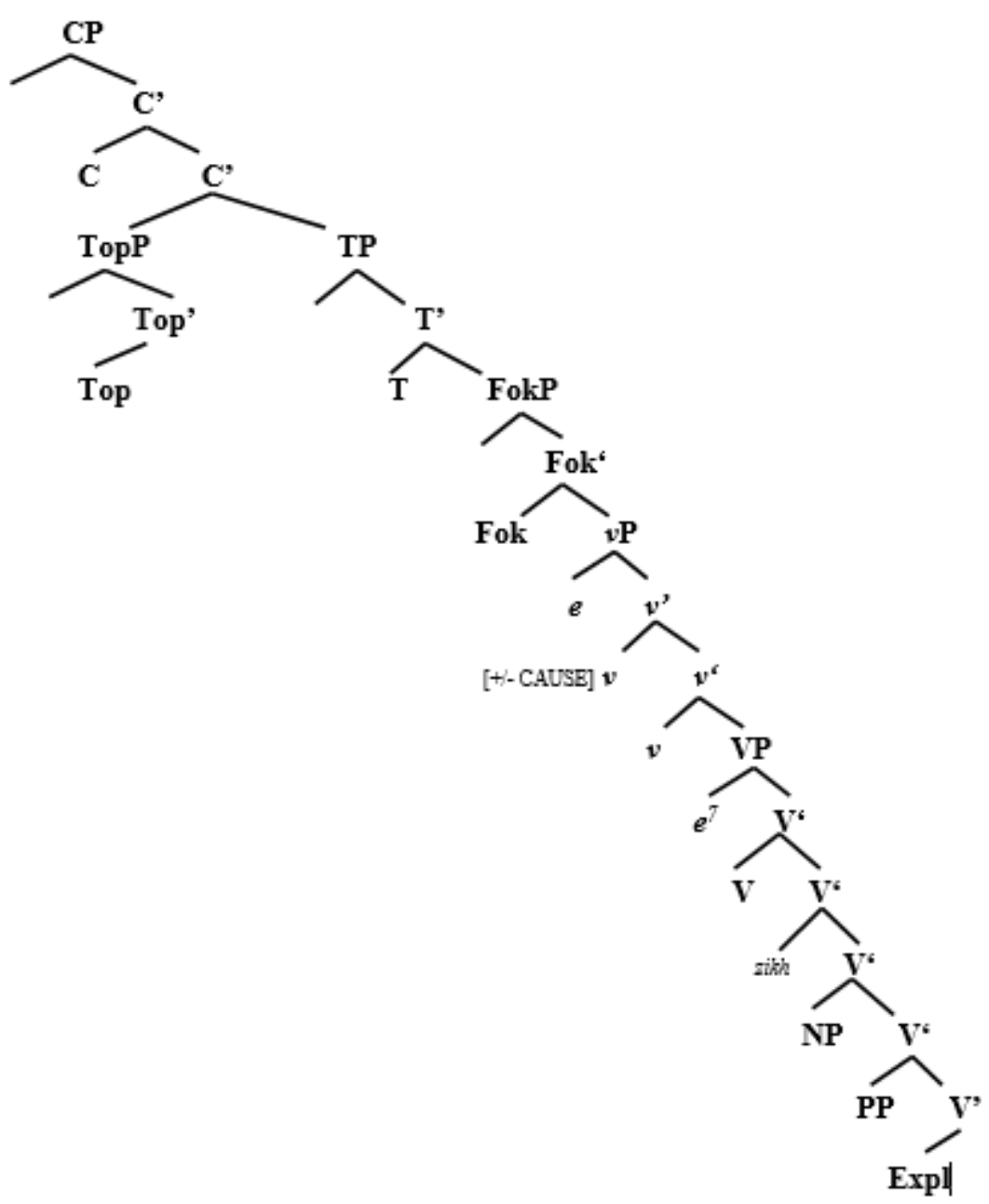

(1b)

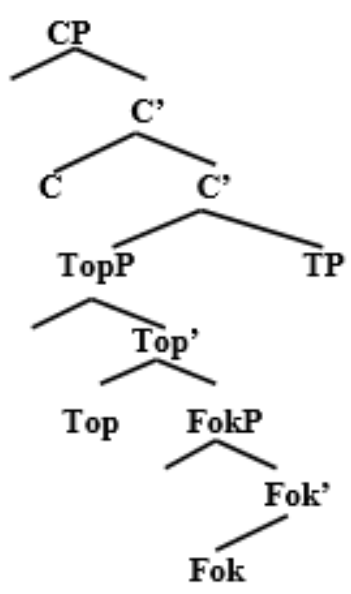

Das Modell (1a) bezieht sich auf das analysierte syntaktische Phänomen, in dem die TopikProjektion zwischen C und TP aktiviert ist. Die tief liegende VP enthält eine verfügbare Komplement-NP mit der zugewiesenen thematischen Rolle Patiens/Thema. Der Spezifizierer

\footnotetext{
6 e steht für empty (,leer').
} 
von VP ist leer. Eine adverbiale PP oder ein Expletiv sind frei verfügbar. In der Projektion $v \mathrm{P}$ werden unakkusativische Verben [- CAUSE] aktiviert, wenn der Spezifizierer von VP leer ist.

FokP (Fokus) im satzinternen Bereich ist eine funktionale Projektion (cf. Belletti 1999) ${ }^{7}$. Wenn im Spezifizierer von FokP eine Nominalphrase und im Kopf ein Verb stehen, kann der Nominativ zugewiesen und Kongruenzmerkmale überprüft werden, so dass Agree stattfindet. Es wird dementsprechend angenommen, dass Nominativzuweisung und Merkmalüberprüfung in der Relation Spez-Kopf von FokP erfolgen, (cf. Kapitel 5.1). Ein sichtbarer Effekt dieser Prozedur ist die Fokussierung einer Subjekt-NP.

Andererseits ist aber ersichtlich, dass der TopP-Bereich der C-Domäne und der TP-Bereich inklusive FokP in der C-Command-Relation stehen. Dies kann zur Folge haben, dass die CDomäne auf den tiefen TP-Bereich sensitiv ist. Wenn in dem tiefen FokP-Bereich (1a) Kasuszuweisung und Merkmalüberprüfung stattfinden, ist der Fokus-Bereich der C-Domäne (1b) nicht aktiviert und eine unmarkierte Topik-Projektion weiterhin verfügbar. Für das analysierte Phänomen des Jiddischen wurde das Konzept von Rizzi $(1997,2004)$ und Belletti $(1999,2004)$ in der Weise modifiziert, dass die C-Domäne nur eine Topik-Projektion enthält und in dem satzinternen Bereich zwischen dem TP- und dem vP-Bereich eine FokusProjektion situiert ist. Fokus-Projektionen in der C-Domäne und Topik-Projektionen im tiefen satzinternen Bereich werden hier nicht analysiert. Es ist allerdings möglich, dass (1b) als Darstellung einer Topikalisierung genutzt werden kann, in der eine XP im Fokus der CDomäne markiert wird.

\section{$3 \quad$ Problemstellung}

Die satzfinale Subjektposition im Jiddischen wurde bereits in Analysen von Prince beobachtet (cf. 1989, 1993):

(2) es geyt epes in vald a yid (Prince 1993: 176)

es geht irgendein ${ }^{8}$ im-wald-Dat ein-jude-Nom

Ein Jude scheint im Wald spazieren zu gehen.

Prince (1993: 176) spezifiziert hier allerdings weder Verbgruppe noch thematische Rolle von Subjekt. In ihren Beispielsätzen scheinen pragmatische Regelung und Akzentsetzung im Vordergrund zu stehen. Auch Kongruenzrelationen eines hinten stehenden Subjekts bleiben unklar.

Das Problem der Merkmalüberprüfung eines postverbalen Subjekts wurde im Minimalischen Programm bereits diskutiert ${ }^{9}$. Im Jiddischen kann allerdings die folgende eigenartige Strukturierung beobachtet werden:

\footnotetext{
${ }^{7}$ Es wird hier die Idee von Belletti (1999) übernommen und in der Weise modifiziert, dass im satzinternen Bereich eine Fokusprojektion berücksichtigt ist.

8 Ohne erkennbaren Grund. Den Hinweis verdanke ich Marion Aptroot (pers. Mitteilung).

${ }^{9}$ In Chomsky (1995) wird die Merkmalüberprüfung eines postverbalen Subjekts anhand von folgendem Beispielsatz diskutiert:

a. there is $[\alpha$ a strange man] in the garden

b. there seems to [ $\alpha$ a strange man] [that it is raining outside] (Chomsky 1995: 200)
} 
(3)

\title{
אין הימל האָט זיך אָפגעריסן אַ שטערן [...]
}

\author{
(I. Bashevis Singer 1931)
}

in himl hot zikh opgerisn a shtern

im-himmel-Dat hat sich abgerissen ein-stern-Nom

Vom Himmel hat sich ein Stern losgerissen.

In (3) wird die satzfinale Nominativ-NP nicht als Agens, sondern als Patiens/Thema interpretiert. Diese Phrase ist fokussiert. Man kann zugleich beobachten, dass die präverbal stehende PP unmarkiert ist (wie dies im Modell 1a angenommen wird). Die Möglichkeit einer kontrastiven pragmatischen Akzentsetzung $(1 b)^{10}$ wird hier außer Acht gelassen.

Eine andere mögliche Konfiguration, die im folgenden Aufsatz nicht analysiert wird, entsteht bei kausativen transitiven Verben mit satzfinaler Subjektposition. Entweder Subjekt (4a) oder Objekt (4b) werden durch eine entsprechende Akzentsetzung markiert, cf.

a. dos bukh hot geshribn ODEM

das buch-Akk hat geschrieben Adam-Nom

Das Buch hat ADAM geschrieben.

b. dos BUKH hot geshribn Odem

das buch-Akk hat geschrieben Adam-Nom

Das BUCH hat Adam geschrieben.

Im vorliegenden Aufsatz wird versucht, die Strukturierung vom Typ (3) zu analysieren, in der das Subjekt satzfinal platziert ist und den Satzkern unakkusativische Verben formen. Den Ausgangspunkt der Analyse bilden Sätze mit präverbal stehenden unmarkierten PPs, aber auch Sätze mit dem Expletiv es und alternativ mit der Verbvoranstellung. Es wird angenommen, dass bei Derivationen analysierter Sätze ein eigenständiger Mechanismus aktiv ist, in dem sich mehrere Elemente verzahnen: ein unakkusativisches Verb mit einer NP, die thematische Rolle der NP, die Position der NP im Satz, Informationsstruktur Topik/Fokus und deren Korrelation. Es können dazu mehrere Fragen gestellt werden: wie kommen Nominativzuweisung und Kongruenzrelationen zustande, wenn die Subjekt-NP so tief platziert ist? Kann die satzfinale Position der NP als ein Argument für VO im Jiddischen betrachtet werden? Muss für das Jiddische neben dem expletiven es auch ein Nullexpletiv

\footnotetext{
In (a) ist $\alpha$ nicht in der richtigen Position, um Kasus zu überprüfen, da dies in Spez von Agr $_{\mathrm{s}}$ oder in Spez von T geschehen muss (gemäß EPP/Extended Projection Principle, cf. die ausführliche Diskussion in Mohr 2002). (a) erfolgt im LF-Bereich, wenn $\alpha$ in die Matrixposition angehoben wird, wo zugleich das Expletiv beigefügt wird. Die Anhebung hinterlässt eine Spur $t$. Das Matrixsubjekt ist [ $\alpha$-there] auf LF. In der Matrixposition werden sämtliche Merkmale überprüft, aber interpretierbare Merkmale bleiben nur in der Spur $t$ zurück (im assoziierten Element $\alpha$ ), wo eine Kette $(\alpha, t)$ entsteht. Im Unterschied dazu wird der Kasus von $\alpha$ in (b) in der Präpositionalphrase zugewiesen, so dass there in diesem Fall frei beigefügt ist (cf. Chomsky 1995: 200-201). In neueren Ansätzen wird postuliert, dass die Kongruenzrelation Agree auch aus einer Entfernung zustande kommen kann (Long-Distance Agree), während die betreffende NP/DP nicht angehoben wird und in situ bleibt (cf. u. a. Boeckx 2009).

${ }^{10}$ Es ist annehmbar, dass die topikalisierte Phrase in der Fokus-Projektion der C-Domäne platziert ist und daher den Kontrastakzent trägt.
} 
angenommen werden, wenn deklarative V1-Sätze möglich sind? Wie kann die Topik/FokusKorrelation erklärt werden? Sind Ökonomieprinzipien für die beobachtete Strukturierung des Jiddischen verantwortlich?

\section{V1, V2, satzinitiale Elemente und Informationsstruktur}

Um Aspekte einer satzfinalen Subjektposition kennenzulernen, braucht man einen Einblick in die V-Struktur mit präverbal stehenden Elementen und Topik/Fokus-Relationen. Wie man annimmt, ist Jiddisch eine V2-Sprache (cf. u. a. Diesing 2005; Jacobs 2005: 223-226; Kühnert/Wagner 2014). In der satzinitialen Position können (5) ein pronominales Subjekt, (6) eine Subjekt-NP/DP (7) eine Akkusativ-NP ${ }^{11}$ oder (8) eine Wh-Phrase stehen:

כ'האָב שוין צו זיי קיין כוח נישט!

(I. Bashevis Singer 1931)

ikh hob shoyn tsu zey ka koyekh nisht

ich-Nom habe schon zu-sie-Dat keine-kraft-Akk nicht-Satzneg

Ich habe keine Geduld mehr mit ihnen.

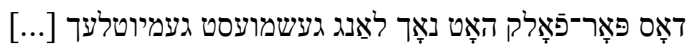

(Forverts 05.03.2013)

dos porfolk hot nokh lang geshmuest gemyutlekh ${ }^{12}$

das-ehepaar-Nom hat noch lange sich unterhalten gemütlich

Das Ehepaar hat sich noch lange gemütlich unterhalten.

דעם 28סטן יולי איז אַקוראַט אויסגעפֵַאלן 99 יאָר זינט עם איז אויסגעברָכן די ערשטע וועלט־מלחמה.

(Forverts 01.08.2013)

dem 28sten yuli iz akurat oysgefaln 99 yor zint es iz oysgebrokhn di ershte veltmilkhome

den-28sten-juli-Akk ist gerade ausgefallen 99-jahr-Nom seit-Konj es-Expl ist ausgebrochen der-erste-weltkrieg-Nom

Am 28. Juli jährt sich zum 99. Mal der Tag, seit der erste Weltkrieg ausgebrochen ist.

\section{Aspekte der satzfinalen Subjektposition}

In der Syntax des Jiddischen kann die folgende Strukturierung beobachtet werden. Den Kern der analysierten Konstruktionen bilden unakkusativische Verben mit einer verfügbaren NP, die am Ende der Derivation die satzfinale Position einnimmt. Unakkusativische Verben umfassen zwei Subgruppen. Eine Subgruppe kann von transitiven Verben „X verursacht $\mathrm{Y}^{\text {“ }}$ abgeleitet werden. Charakteristisch für diese Gruppe ist das Element zikh, das entweder als

\footnotetext{
${ }^{11}$ Generell sind hier satzinitiale Objekte oder Adverbiale möglich.

12 Marion Aptroot (pers. Mitteilung) weist darauf hin, dass dies eine unübliche Form ist, wodurch wahrscheinlich Komik erreicht werden soll. Die korrekte Form ist געמיטלעך / gemitlekh.
} 
formaler Marker einer nicht-kausativen Lesart verwendet wird oder auf einen unbestimmten Experiencer mit Merkmalen [Hum, Indef] bezogen werden kann. Eine andere Subgruppe

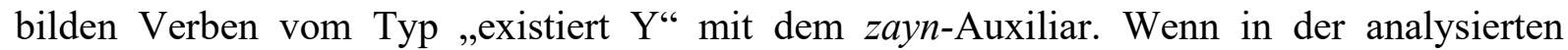
Strukturierung eine adverbiale PP (oder ein Adverbialsatz) verfügbar ist, hat sie eine unmarkierte Position am Satzanfang. Ist kein derartiges Element verfügbar, so wird das Expletiv es aktiviert oder alternativ die Verbanhebung in die C-Domäne. Im Folgenden werden diese Möglichkeiten analysiert.

\subsection{PP im Topik, zikh-Elemente und Fokussierung von Subjekt}

Wie bereits (3) - in himl hot zikh operisn a shtern - zeigt, aber im Unterschied zu (14b), wo eine topikalisierte XP markiert ist, können folgende Beispielsätze genannt werden:

פַון אונטער'ן דאַך עפענט זיך אַ קליין פענסטערל [...]

(Peretz 1920)

fun untern dakh efent zikh a kleyn fensterl

von-unterem-dach-Dat öffnet sich ein-klein-fensterlein-Nom

Von unterem Dach öffnet sich ein kleines Fenster.

b. אויף די אָפגעשניטענע פַעלדער האָבן זיך העל אָפגעצייכנט קופעם היי.

I. Bashevis Singer (1931)

af di opgeshnitene felder hobn zikh hel opgetseykhnt kupes hey

auf-die-abgeschnittene-felder-Dat.Pl haben sich hell abgezeichnet haufen-heuNom

Auf den abgeschnittenen Feldern haben sich Heuhaufen hell abgezeichnet.

Der Versuch, das Phänomen der oben dargestellten Strukturierung im Jiddischen zu erklären, geht auf das Konzept der Unakkusativität (Perlmutter 1978) und das Konzept der Generalisierung von Burzio ${ }^{13}$ (1986) zurück. In (3) und (15) kann beobachtet werden, dass das satzfinale Subjekt von zikh-Verben begleitet wird. Man kann zu diesen Verben, vergleichbar zu dem Deutschen, transitive Varianten finden: (3) X reißt Y ab; (15a) - X öffnet $\mathrm{Y},(15 \mathrm{~b})$ - X zeichnet $\mathrm{Y} a b$.

Es ist in diesen Sätzen außerdem Folgendes ersichtlich: Nominativ-NPs können nicht als Agens interpretiert werden und die Verben sind nicht kausativ. Wenn transitive Verben und $z i k h$-Varianten aus einem homogenen Modell abgeleitet werden, kann zikh für die Verbgruppe vom Typ (15) als ein funktionales Element betrachtet werden, das kausative Konstruktionen blockiert.

13 Das Verb, das dem Subjekt die thematische Rolle zuweist, weist auch den Objektkasus zu. 
(16)

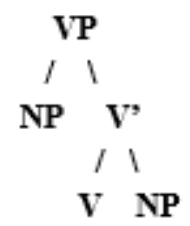

(17)

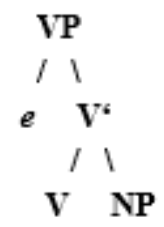

(16) ist ein transitives Verb. In (17) ist SpezVP leer (e). Als Konsequenz kann eine NP mit der thematischen Rolle Patiens/Thema in der Kopf-Komplement-Relation (V NP) keinen Objektkasus zugewiesen bekommen (Burzios Generalisierung). In dieser Konfiguration kann das Merkmal [+CAUSE] nicht aktiviert werden.

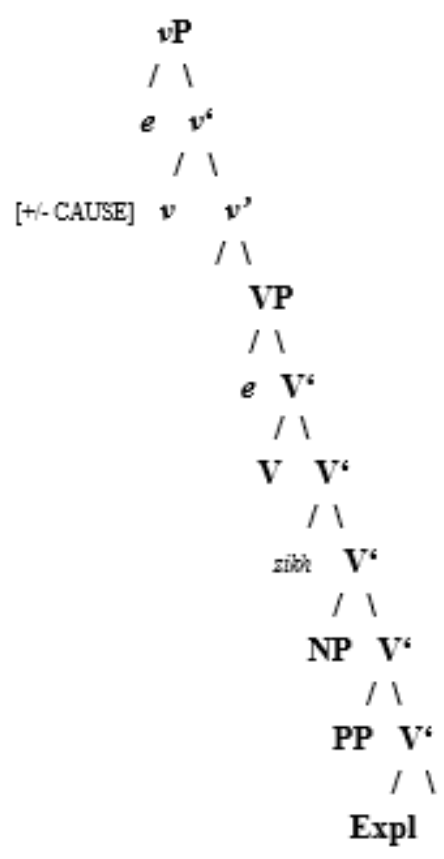

Da die VP nicht vollständig ist (eine defekte VP), erreicht eine V-Anhebung in den $v \mathrm{P}-$ Bereich das Merkmal [- CAUSE]. Eine parallele zikh-Anhebung blockiert die kausative Lesart.

Aus den Sätzen (3) in himl hot zikh operisn a shtern, (15ab) resultiert, dass dem jiddischen Satz jeweils zikh beigefügt ist. Der oben beschriebenen Strukturierung scheint ein genereller Mechanismus zugrunde zu liegen. Als Auslöser kann dabei ein Sachverhalt angenommen werden, in dem ein Geschehen keinen Urheber hat. Dieser Sachverhalt muss in eine syntaktische Struktur umgeformt werden. Zur Verfügung stehen allerdings zweistellige Verben vom Typ „X efent Y“/,X öffnet Y“, wobei X keine semantische Interpretation hat. 
Als Konsequenz werden syntaktische Strukturen wie (3), (15) gebildet. Als formale Blockade einer kausativen Lesart kann folglich zikh betrachtet werden, das ein Kompensationselement für den absorbierten Akkusativ ist. Zikh ist nicht anaphorisch interpretierbar. Es entsteht aber auf LF ein pseudoreflexiver Anaphereffekt, der dem Subjekt einen illusorischen Urheberstatus gibt. Man muss darauf hinweisen, dass (3) und (15) nur dann wohlformt sind, wenn die satzfinale NP einen nominalen Kopf hat. Die Derivation scheitert bei Pronominalisierungen (19b), cf. den Satz (3), der als (19) wiederholt wird:

(19) a. in himl hot zikh opgerisn a shtern

b. in himl hot zikh opgerisn *er

c. in himl hot er zikh opgerisn

d. in himl hot zikh er opgerisn

Anhand von (19) kann angenommen werden, dass die Nominativzuweisung und Merkmalüberprüfung in verschiedenen Bereichen stattfinden können. Wenn die Projektion keinen nominalen Kopf hat, kann die Merkmalüberprüfung im FokP-Bereich nicht stattfinden und die Derivation scheitert (19b). Der Überprüfungsmechanismus scheint in (19a) entlastet zu sein, da bei nominalen Köpfen die 3. Person automatisch selegiert ist und eine Art Default-Strategie genutzt werden kann. Genus der NP hat keinen beobachtbaren Kongruenzeffekt im verbalen Bereich. Es kann angenommen werden, dass Genus in Bezug auf analysierte NPs in FokP geprüft und gelöscht wird. Nach der Überprüfung bleibt nur Numerus interpretierbar. Wenn die Kasuszuweisung und Merkmalüberprüfung im FokPBereich erfolgen, braucht die NP nicht weiter bewegt zu werden. (19c), (19d) weisen darauf hin, dass bei Pronominalisierungen weitere Bewegungen notwendig sind und die Nominativzuweisung wie auch Merkmalüberprüfung im TP-Bereich stattfinden. (19a) hat allerdings eine Alternative in (20):

(20) a shtern hot zikh opgerisn in himl

Das Subjekt in (20) ist - im Unterschied zu (19a) - unmarkiert. Die Nominativzuweisung und Merkmalüberprüfung finden im TP-Bereich regelrecht statt.

In Anlehnung an Rizzi (1997, 2004) und Belletti (1999), aber mit Modifikationen in Bezug auf das Jiddische kann die folgende Kartographie des analysierten Mechanismus angenommen werden: 
(21)

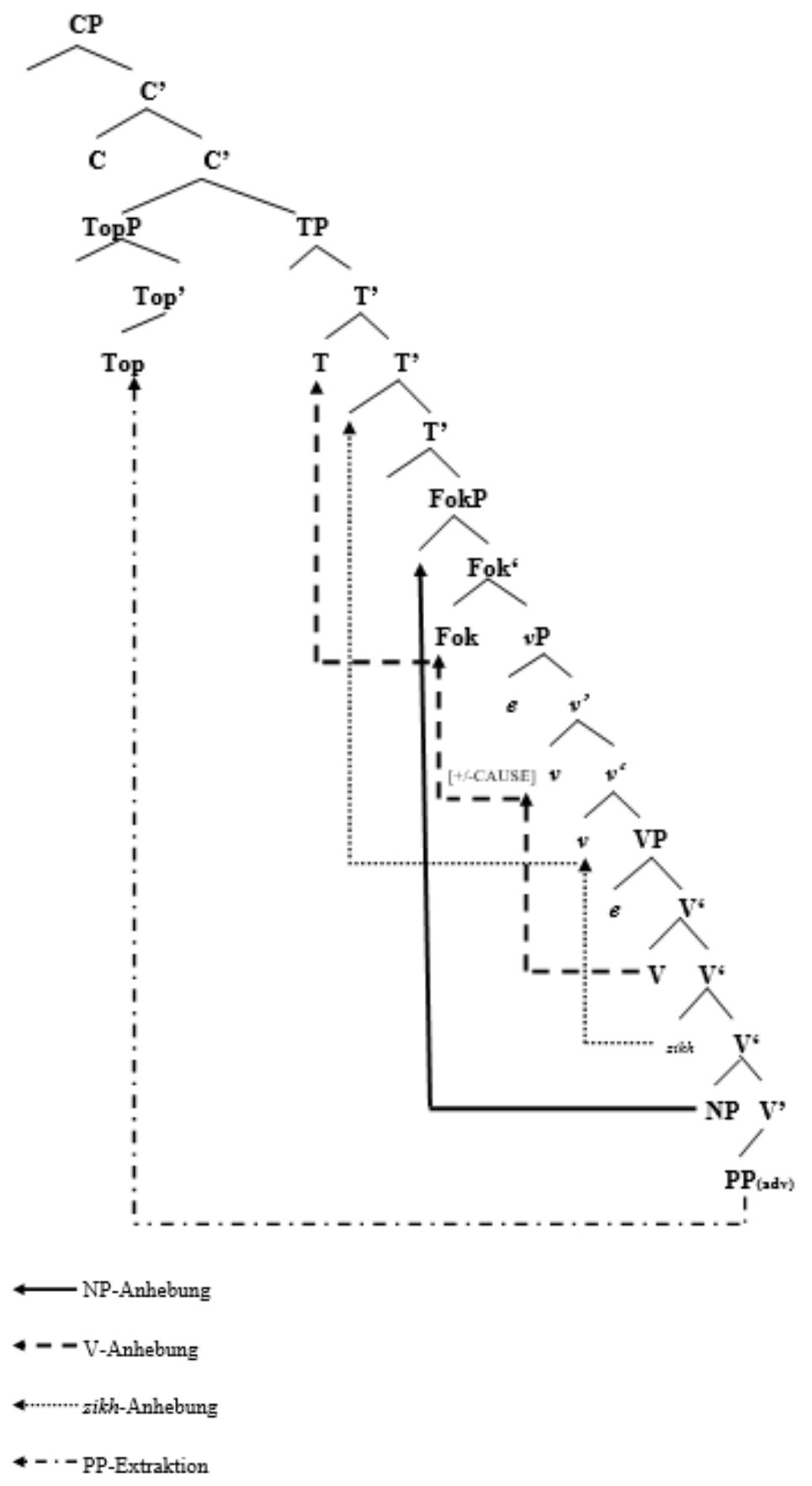


(22)

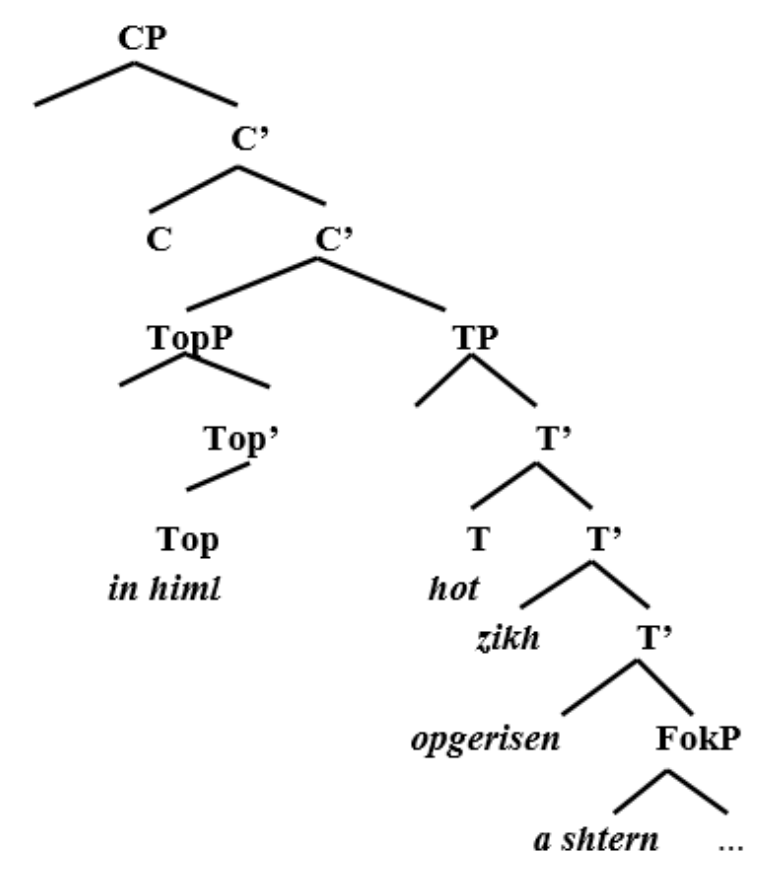

In (21) ist der Topik-Bereich zwischen $\mathrm{C}$ und dem TP-Bereich aktiviert. Als Effekt der Derivation kann die Fokussierung von Subjekt betrachtet werden, daher findet die NPAnhebung in den Spezifizierer von FokP statt. Belletti (1999) nimmt an, dass FokP als funktionale Projektion auch für die Kasuszuweisung/Merkmalüberprüfung genutzt werden kann. In Belletti (2004: 25) gibt sie allerdings diese Idee zugunsten einer entfernten Kasusüberprüfung nach.

Man kann annehmen, dass das analysierte Phänomen im Jiddischen für die erste Variante, also für die Kasuszuweisung/ Merkmalüberprüfung im lokalen Spez-Kopf-Bereich von FokP stärker spricht, da FokP die nächstliegende Projektion ist, die funktional genutzt werden kann. Die gesamte Operation ist dadurch sparsamer. Der Effekt der Fokussierung von Subjekt wird infolge der erreichten Strukturierung sichtbar und die NP braucht nicht höher bewegt zu werden. Die entfernte Kasuszuweisung und Merkmalüberprüfung ist schwieriger nachweisbar, obwohl sie konzeptuell möglich ist. Diese Idee wird hier nicht weiter verfolgt.

Wenn die NP-Anhebung in den FokP-Bereich stattfindet, wird das Verb zunächst in die $v$ Projektion bewegt, um [+/- CAUSE] zu selegieren. Parallel mit dem Verb ist im vP-Bereich $z i k h$ platziert. In dieser Konfiguration und mit einer vakanten agentiven NP wird für das Verb das Merkmal [- CAUSE] selegiert. Da FokP als funktionale Projektion betrachtet wird, kann in deren Spezifizierer der Nominativ zugewiesen und dadurch eine NP zum Subjekt werden. Diese Markierung zieht das Verb in den funktionalen FokP-Bereich an, wo Kongruenzmerkmale überprüft werden. Wie bereits angenommen wurde, hat nur Numerus einen beobachtbaren Kongruenzeffekt. Da FokP die nächstliegende funktional verfügbare Projektion ist und interpretierbare Merkmale minimalisiert sind, ist dadurch der Überprüfungsmechanismus in FokP sparsam.

Die Top-Projektion in der C-Domäne ist auf die Positionierung von Subjekt sensitiv, so 
wird dieser Bereich nach der Kasuszuweisung und Merkmalüberprüfung in FokP für andere Elemente frei. Um Ambiguitäten zu vermeiden, wird die PP aus dem tiefen VP-Bereich extrahiert und in den Kopf von TopP der C-Domäne platziert. Im verzweigten TP-Bereich erreicht das Verb seine Zielposition. Zikh folgt dem Finitum und wird adjazent platziert. (22) illustriert Zielpositionen von lexikalischen Elementen im analysierten Mechanismus.

Im Unterschied zu der Analyse vom Typ (3) in himl hot zikh opgerisn a shtern, in der zikh nur als funktionales Element dient, kann in (23), (24) zikh als Experiencer interpretiert werden.

פַון ערגעצ האָט זיך געהערט דאָס קרעכצען פַון אַ סָָווע.

\section{Bashevis Singer 1931}

fun ergets hot zikh gehert dos krekhtsn fun a sove

von irgendwoher hat sich gehört das-kreischen-Nom von-einem-uhu-Dat

Von irgendwoher hat man das Kreischen von einem Uhu hören können.

פَון קעסל ארויס האָט זיך געהערט אַ פַאַרשטיקטער מענטשלעכער קוויטש.

\section{Bashevis Singer 1931}

fun kesl aroys hot zikh gehert a farshtikter mentshlekher kvitsh

von-kessel-Dat heraus hat sich gehört ein-verstickter-menschlicher-quicken-Nom

Von einem Kessel heraus hat man ein verstummtes menschliches Quicken hören können.

Es ist ersichtlich, dass zikh in (23), (24) in der Struktur von Wahrnehmungsverben die Merkmale [Hum, Indef] hat. Man kann dies wie folgt darstellen:

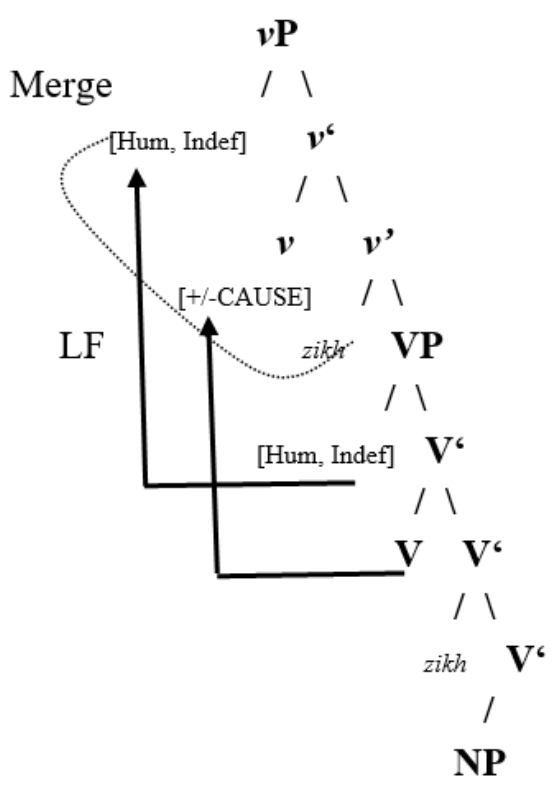


Die VP ist insofern defekt, als es keine NP für den Experiencer gibt. Die V-Anhebung in den $v \mathrm{P}$-Bereich hat zur Folge, dass zikh beigefügt wird. Die Bewegung von Merkmalen [Hum, Indef] in die SpezPosition von $v \mathrm{P}$ kann nur auf LF erfolgen. In dieser Konfiguration findet Merge statt. Das Element zikh bekommt die Interpretation [Hum, Indef] zugewiesen.

\subsection{Verben mit dem zayn-Auxiliar}

Eine Subgruppe von unakkusativischen Verben bilden Intransitiva, die über eine NP verfügen, aber nicht wie in 5.1 von transitiven Verben „X verursacht $\mathrm{Y}^{\prime}$ abgeleitet werden können. Die thematische Rolle dieser NP ist ebenfalls Patiens/Thema. Da nicht mehr eine blockierende Funktion in der gegebenen Strukturierung notwendig ist, ist auch zikh nicht verfügbar. Ein distinktives Element für diese Subgruppe ist das zayn-Auxiliar. Genauso wie in 5.1 kann auch hier eine unmarkierte topikale PP stehen:

איבערן דיל זיינען געקראָן ווערים [...]

(I. Bashevis Singer 1931)

ibern dil zaynen gekrokhn verim

über-diele-Dat sind gekrochen würmer-Nom

Über die Diele sind Würmer ${ }^{14}$ gekrochen.

$$
\text { ערגעץ ווייט פַארן בליק זיינען אויפגעגאנגגען פלעמלעך ווי הבדלות [...] }
$$

\section{Bashevis Singer 1931}

ergets vayt farn blik zaynen ufgegangen flemlekh vi havdoles

irgendwo weit vor-blick-Dat sind aufgegangen flammen-Nom wie hawdalas

Irgendwo weit vor dem Blick sind Flammen aufgegangen wie Hawdalas.

Sätze (26) und (27) haben eine existentiale Lesart „existiert Y“. Wenn eine satzfinale NP nicht als Agens interpretiert werden kann, kann auch hier angenommen werden, dass der Spezifizierer im VP ebenfalls leer ist und die NP im internen VP-Bereich basisgeneriert wird.

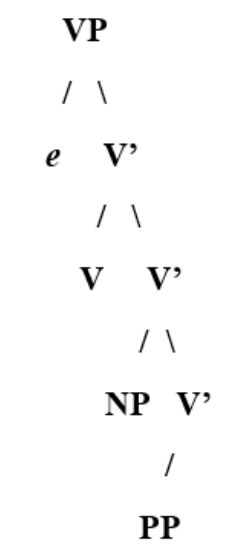

\footnotetext{
${ }^{14}$ Es wird angenommen, dass hier Würmer nicht als Agens zu verstehen sind, sondern dass sich der Sachverhalt auf die Existenz von Würmern bezieht (im Sinne es gibt Würmer).
} 
Gleichwertig wie PPs können auch im CP-Bereich stehende eingebettete Sätze betrachtet werden.

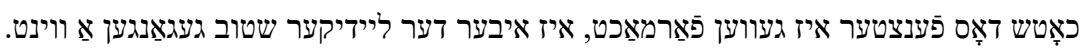

(I. Bashevis Singer 1931)

khotsh dos fentster iz geven farmakht iz iber der leydiker shtub gegangen a vint obwohl das-fenster-Nom ist gewesen zugemacht ist über der-leerer-stube-Dat gegangen ein-wind-Nom

Obwohl das Fenster geschlossen war, ist über die leere Stube der Wind gegangen.

Es kann angenommen werden, dass auch hier eine finale NP in die SpezPosition von FokP angehoben werden muss, um den Nominativ zugewiesen zu bekommen. Das Verb wird in den Kopf von FokP bewegt, so dass die Merkmalüberprüfung stattfinden kann.

\subsection{Expletivmarker ${ }^{15}$}

Bis jetzt wurden Sätze dargestellt, in denen eine frei verfügbare PP enthalten war, bzw. ein Satz (29) hätte beigefügt werden können. Im Jiddischen sind allerdings noch weitere Alternativen möglich. Die Literatur enthält Analysen des jiddischen Expletivs es, cf. u. a. Prince (1989), Diesing (2005). Im Korpus können auch Belege mit der satzfinalen Subjektposition und dem Expletiv es gefunden werden:

ס'האָט זיך געהערט אַ פַארשטיקט שפילן [...]

(I. Bashevis Singer 1931)

s'hot zikh gehert a farshtikt shpiln

es-Expl hat sich gehört ein-dumpf-spielen-Nom

Es wurde ein dumpfes Spielen gehört.

b.

עם זיינען אומגעקומען בסך־הכל 10 מיליאָן מיליטער־לייט [.]

(Forverts 01.08.2013)

es zaynen umgekumen besakhakl 10 milyon militerlayt

es-Expl sind umgekommen insgesamt 10-millionen-soldaten-Nom

Es sind insgesamt 10 Millionen Soldaten umgekommen.

Man sieht, dass das Expletiv es sowohl bei unakkusativischen Verben mit zikh als auch mit dem zayn-Auxiliar vorkommen kann. In subordinierenden Relationen bleibt präverbales expletives es auch erhalten:

(31) a.

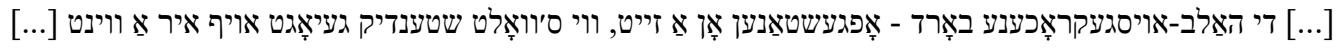

(I. Bashevis Singer 1931)

di halb oysgekrokhene bord opgeshtanen on a zayt vi's volt shtendik geyogt af ir

\footnotetext{
${ }^{15}$ Als expletives es wird hier nur das Element betrachtet, das in der präverbalen Position steht und mit der Subjekt-NP korreliert ist.
} 
a vint

die-halb-ausgekrochene-bard-Nom abgestanden an-eine-seite-Dat wie-Konj es wollt ständig gejagt auf-ihr-Dat ein-wind-Nom

[...] der halb ausgefallene und auf einer Seite abstehende Bard, als ob auf ihm ständig der Wind gejagt hätte [...]

b. [....] און צו קינדער, וואָס ס'איז זיי פלוצלונג אָפגענומען געווארן דער דיבור.

(I. Bashevis Singer 1931)

un tsu kinder vos s'iz zey plutslung opgenumen gevorn der dibur

und zu-kinder-Dat was-Rel es-Expl sie-Dat plötzlich abgenommen geworden die-sprache-Nom

[...] und zu Kindern, die plötzlich die Sprache verloren haben.

In Anlehnung an Mark (1978) zeigt Jacobs (2005: 226), dass Expletive lediglich in einer Korrelation mit NP-Subjekten möglich sind, und mit Pronomina sind sie nicht erlaubt:

(32) a. Es tut mir vej der fus ${ }^{16}$.

b. es iz gəkumən der lerər

c. $\quad *$ es iz er gəkumən (Jacobs 2005: 226)

Es kann angenommen werden, dass das Expletiv bereits in der VP enthalten ist:

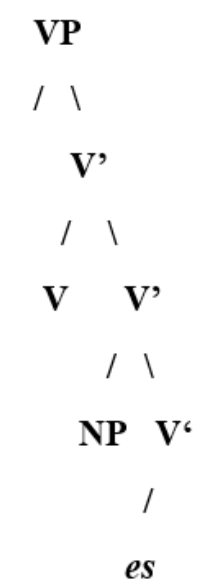

Das Expletiv es ist aktiv, wenn die NP in die SpezPosition von FokP angehoben wird und kein frei verfügbares Element in die C-Domäne bewegt werden kann. Bei der Anhebung der NP in die SpezPosition von TP und die Platzierung vor dem Finitum erfolgt seine Deaktivierung. Bei der Verbvoranstellung wird das Expletiv es ebenfalls deaktiviert (cf. 5.4).

\footnotetext{
16 Bei Jacobs (2005: 226) Beispielsatz (11). Transliteration und Orthographie nach Jacobs.
} 


\subsection{Verbvoranstellung}

In deklarativen Sätzen des Jiddischen ist auch eine Verbvoranstellung möglich, die als Konkurrenzstruktur zu unmarkierten XPs und dem Expletiv es betrachtet werden kann. Das Problem, ob diesen Konstruktionen eine Verbbewegung in die C-Domäne - wie dies bei PPs und dem Expletiv es der Fall war - zugrunde liegt oder, ob ein Nullexpletiv angenommen werden muss, bleibt hier offen. Wenn das Schema (1a) für die Analyse des Jiddischen korrekt ist, muss eine Nullexpletiv-Annahme, zumindest für analysierte Konstruktionstypen, in Frage gestellt werden. Es kann beobachtet werden, dass vorangestellte Verben einen Topik-Effekt haben (im Sinne von Aboutness-Konzept), daher scheint eine Verbbewegung in den TopPBereich der C-Domäne eine bessere Begründung $\mathrm{zu}$ sein als die Annahme eines Nullexpletivs.

האָט זיך געפירט צווישן אים און דעם פָאָטער אַ מלחמה.

(Bimko 1921)

hot zikh gefirt tsvishn em un dem foter a milkhome

hat sich geführt zwischen ihm-Dat und dem-vater-Dat ein-krieg-Nom

Es wurde zwischen ihm und dem Vater ein Krieg geführt.

b. זענען אָבער אויף דער וועלט פَאַראַן אַזוי פַיעל נאַרישע און שלעכטע פּראוועם.

(Peretz, ed.1920)

zenen ober af der velt faran azoy fil narishe un shlekhte praves

sind aber auf-der-welt-Dat zugänglich solch dumme-und-schlechte-sitten-Nom

Es gibt aber auf der Welt viele dumme und schlechte Sitten.

(34) zeigt, dass Verbvoranstellung und finale Subjektposition eine eigenständige syntaktische Variante des Jiddischen bilden.

\section{$6 \quad$ Fazit}

Empirische Daten des Jiddischen zeigen, dass eine satzfinale Subjektposition, unakkusativische Verben und Topik/Fokus-Relationen von einem homogenen syntaktischen Mechanismus umspannt werden. Wenn in der Struktur der Verbalphrase nur eine NP/DP verfügbar ist, die die Patiens/Thema-Rolle trägt, kann diese NP gemäß Burzios Generalisierung den Akkusativ nicht durch die Verbrektion zugewiesen bekommen. Wie kartographische Ansätze postulieren (Rizzi 1997, 2004), werden Topik und Fokus als Köpfe funktionaler Projektionen in die Syntax integriert. Belletti (1999) schlägt vor, dass Fokus auch eine tiefere funktionale Projektion hat, in deren Spez-Kopf-Position der Nominativ zugewiesen und Kongruenzmerkmale überprüft werden können und demzufolge die Operation Agree stattfinden kann. Dies wird mit entsprechenden Modifikationen für das Jiddische übernommen.

Es wird angenommen, dass die NP eines unakkusativischen Verbs nicht in den TP-Bereich angehoben werden muss, um den Kasus zugewiesen zu bekommen. Wenn die Fokussierung der Phrase als ein sichtbarer Effekt der Derivation betrachtet wird, kann die Fokus-Projektion 
funktional genutzt werden. Nimmt man an, dass die betreffende NP ein basisgeneriertes Objekt und die kanonische Folge VO ist, führt die optimale Anhebung der NP in den Spezifizierer der Fokus-Projektion, um den Nominativ zugewiesen zu bekommen. Diese Anhebung und die Aktivierung der Kasuszuweisung ist nur bei Projektionen nominaler Köpfe zulässig. Bei Pronominalisierungen muss die Kasuszuweisung/ Merkmalüberprüfung im TPBereich stattfinden.

Es wird angenommen, dass die Merkmalüberprüfung bei Projektionen nominaler Köpfe - im Unterschied zu Pronominalisierungen - sparsamer verläuft, da die 3. Person automatisch selegiert wird, was eine Art Default-Strategie sein kann. Genus bei NPs analysierter Art ist nicht interpretierbar, es wird daher nach der Überprüfung gelöscht. Das einzige Merkmal, das einen Kongruenzeffekt (Nomen-Verb) hat, ist Numerus.

Wenn die NP den Spezifizierer von FokP erreicht, bekommt sie den Nominativ zugewiesen. Parallel wird das Verb in den Kopf von FokP angezogen. In der Spez-Kopf-Relation werden Person und Genus überprüft und gelöscht. Die Numerus-Überprüfung resultiert in der Selektion von Singular oder Plural für die V-Projektion. Die NP, die zum Subjekt wird, bleibt in FokP zurück, während die V-Projektion ihre Zielposition im TP-Bereich erreicht oder in die C-Domäne bewegt wird, wenn deklaratives V1 beabsichtigt ist.

Die Zielposition von Subjekt in FokP hat Konsequenzen für die ganze Strukturierung analysierter Sätze und deren LF-Interpretation. Da der funktionale Topik-Bereich der CDomäne in der C-Command-Relation zu den tieferen Projektionen steht, ist er auch auf die Subjektposition sensitiv. Die Fokussierung von Subjekt in der tiefen Projektion deaktiviert die Möglichkeit für Fokussierung anderer Elemente, wenn sie in die C-Domäne gelangen. Den in die C-Domäne bewegten Elementen steht nur ein unmarkiertes Topik zur Verfügung. Ist in der Satzstruktur eine adverbiale PP (bzw. Adverbialsatz) verfügbar, so wird sie aus der tiefen Projektion extrahiert und in die C-Domäne angehoben. Wenn keine derartige Phrase verfügbar ist, wird in der C-Domäne das Expletiv es genutzt. Eine alternative Möglichkeit ist die V-Bewegung in die C-Domäne, cf. in himl hot zikh obgerisn a shtern (3), (19), (22) s'hot zikh gehert a farshtikt shpiln (30) - hot zikh gefirt tsvishn em un dem foter a mikhome (34).

Die beobachtete Positionierung in der C-Domäne (i) PP, (ii) es, (iii) V1 eröffnet die Frage, wie das Extended Projection Principle (EPP) im Jiddischen zustande kommt. In (ii) kann zwar angenommen werden, dass das Expletiv es auf eine mögliche Kasuszuweisung und Merkmalüberprüfung in dieser Position hinweist. Aber dann muss in (iii) ein Nullexpletiv angenommen werden, was eine Konkurrenzlösung zu (ii) darstellt. In (i) müsste neben der PP ein Nullexpletiv angenommen werden. Nullexpletive scheinen in analysierten Sätzen problematisch zu sein.

Man kann allerdings das EPP als ein starkes Merkmal der funktionalen Topik-Projektion in der C-Domäne betrachten, das im Spezifizierer von TopP situiert ist, während im Kopf von TopP (i) eine PP, (ii) das Expletiv es oder (iii) ein Verb(komplex) stehen kann. 
(i)

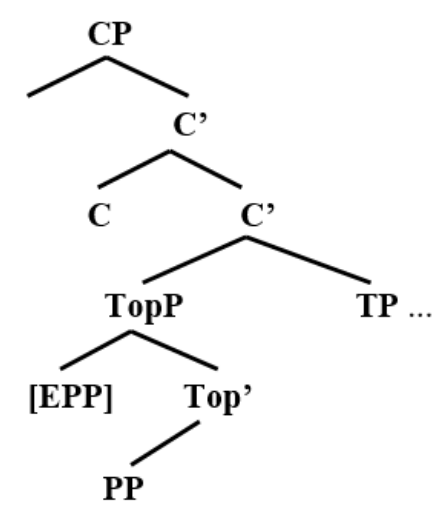

(ii)

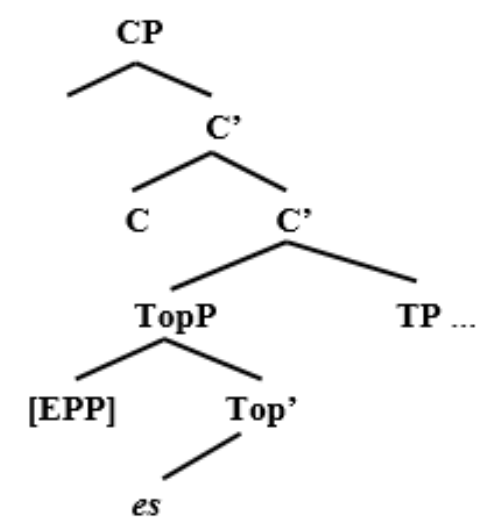

(iii)

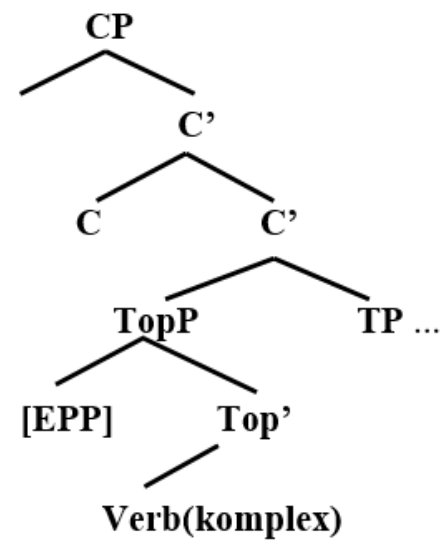

Das EPP ist sensitiv auf TP und auf den tiefen FokP-Bereich, da TopP der C-Domäne und FokP in der C-Command-Relation stehen. Wenn in der Satzstruktur eine PP verfügbar ist, kann sie den Kopf der Topik-Projektion besetzen. Der Unterschied in der Alternative es vs. V1 scheint darin zu liegen, dass das Verb (Verbkomplex) bei der es-Variante im TP-Bereich zurückbleibt, während das Verb bei V1 den TopP der C-Domäne erreicht und als Topik im Sinne von Aboutness-Konzept interpretiert wird.

Die satzfinale Subjektposition kann auch als ein Argument für die VO-Abfolge im Jiddischen betrachtet werden, obwohl analysierte Sätze nur einen Aspekt in der komplexen Strukturierung der jiddischen Syntax darstellen. Es kann allerdings angenommen werden, 
dass die analysierte NP bei Verben vom Typ „X verursacht $\mathrm{Y}^{\prime}$, „,existiert $\mathrm{Y}^{\prime}$ als Objekt basisgeneriert ist. Da aber die Akkusativzuweisung nicht möglich ist (Burzios Generalisierung), scheint eine Bewegung der rettende Weg zu sein. Eine minimale Anhebung führt in die FokP. Da im Deutschen, einer OV-Sprache, nicht erlaubt ist, den Akkusativ oder Nominativ hinter einem Verbteil (Partizip, Infinitiv etc.) zurückzulassen ${ }^{17}$, muss man annehmen, dass sich die jiddische von der deutschen Syntax in dieser Hinsicht unterscheidet.

Die Analyse zeigt, dass der angenommene syntaktische Mechanismus eine homogene Gruppe von unakkusativischen Verben umspannt, die in Subgruppen von zikh-Verben und Verben mit dem zayn-Auxiliar gegliedert werden können. Man kann annehmen, dass die finale Subjektposition in Sätzen mit unakkusativischen Verben syntaktisch geregelt wird. Die erreichte Strukturierung hat eine entsprechende Topik/Fokus-Interpretation auf LF ohne kontrastive Akzentuierung betreffender Satzelemente. Der präsentierte Mechanismus soll von einer pragmatisch geregelten Organisation des Satzes differenziert werden.

\section{Quellenverzeichnis}

Antologye fun der yidisher proze in poyln tsvishn beyde velt-milkhomes (1914-1939), NyuYork 1946. (=Bimko 1921, I. Bashevis Singer 1931).

Di verk fun I.L. Perets. Driter band. Nyu-York 1920. (= Peretz, ed. 1920).

Forverts 01.08.2013

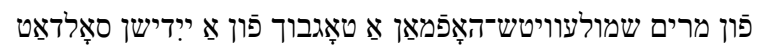

http://yiddish.forward.com/articles/171840/diary-of-a-jewish-soldier/\#ixzz2bI0q2EJu [01.08.2013]

Forverts 05.03.2013

אַ פּינטעלע גוי פַון מרים שמולעוויטש־האָסמאָן

http://yiddish.forward.com/articles/168333/non-jewish-identity/\#ixzz2Mn09mlpg

[05.03.2013]

\section{Literatur}

Abraham, Werner (1992): „Überlegungen zur satzgrammatischen Begründung der Diskursfunktionen Thema und Rhema“. Folia Linguistica 26/1-2: 198-231.

Abraham, Werner (1995): Deutsche Syntax im Sprachenvergleich. Grundlegung einer typologischen Syntax des Deutschen. Tübingen: Narr.

Abraham, Werner/Gelderen van, Elly (eds.) (1995): German: Syntactic Problems Problematic Syntax. Tübingen: Niemeyer.

Aptroot, Marion/Hansen, Björn (eds.) (2014): Yiddish Language Structures. Berlin/Boston: de Gruyter.

Beider, Alexander (2013): "Reapplying the Language Tree Model to the History of Yiddish". Journal of Jewish Languages 1: 77-121.

Belletti, Adriana (1988): "The Case of Unaccusatives”. Linguistic Inquiry 19: 1-34.

Belletti, Adriana (1999): "Inversion as Focalization and Related Questions". Catalan Working Papers in Linguistics 7: 9-45.

\footnotetext{
17 Gisbert Fanselow (pers. Mitteilung) weist darauf hin, dass auch im Deutschen das Subjekt final positioniert sein kann, cf. Gestern ist gefallen ins Wasser ein Stein. Dieses Problem wird hier außer Acht gelassen. Dies kann aber eine breitere Diskussion unter mehreren Aspekten eröffnen, cf. auch Hentschel (2003).
} 
Belletti, Adriana (2004): “Aspects of the Low IP Area”. In: Rizzi, Luigi (ed.): The Structure of CP and IP. The Cartography of Syntactic Structures 2. New York, Oxford University Press:16-51.

Belletti, Adriana/Bennati, Elisa/Sorace, Antonella (2007): "Theoretical and Developmental Issues in the Syntax of Subjects: Evidence from near-Native Italian". Natural language and linguistic theory 25: 657-689.

Beneš, Eduard (1967): „Die funktionale Satzperspektive“. Deutsch als Fremdsprache 1: 2328.

Birzer, Sandra (2014): "Yiddish Passive Constructions". In: Aptroot, Marion/Hansen, Björn (eds.): Yiddish Language Structures. Berlin/Boston, de Gruyter: 269-296.

Boeckx, Cedric (2009): “On Long Distance Agree”. An International Journal of Theoretical Linguistics 1/1: 1-32.

Boeckx, Cedric (ed.) (2011): The Oxford Handbook of Linguistic Minimalism. New York: Oxford University Press.

Burzio, Luigi (1986): Italian Syntax. Dordrecht: Reidel.

Cinque, Guglielmo/Rizzi, Luigi (2008): "The Cartography of Syntactic Structures". Studies in Linguistics. Working Papers 2: 42-58.

Chierchia, Gennaro (2004): "A Semantics for Unaccusatives and its Syntactic Consequences". In: Alexiadou, Artemis/Anagnostopoulou, Elena/Everaert, Martin (eds.): The Unaccusativity Puzzle. Oxford, Oxford University Press: 22-59.

Chomsky, Noam (1995): The Minimalist Program. Cambridge: MIT.

Chomsky, Noam (2000): "Minimalist Inquiries: The Framework". In: Martin, Roger/Michaels, David/Uriagereka, Juan (eds.): Step by Step. Essays on Minimalist Syntax in Honor of Howard Lasnik. Cambridge/Massachusetts, MIT: 89-156.

Chomsky, Noam (2008): “On Phases”. In: Otero, Carlos Peregrin et al. (eds.): Foundational Issues in Linguistic Theory: Essays in Honor of Jean-Roger Vergnaud. Cambridge/Massachusetts, MIT:133-166.

Chomsky, Noam (2013): "Problem of Projections". Lingua 130: 33-49.

Diesing, Molly (1990): "Verb Movement and the Subject Position in Yiddish". Natural Language and Linguistic Theory 8/1: 41-79.

Diesing, Molly (1997): "Yiddish VP Order and the Typology of Object Movement". Natural Language and Linguistic Theory 15/2: 369-427.

Diesing, Molly (2005): “The Upper Functional Domain in Yiddish”. In: Abraham, Werner (ed.): Focus on Germanic Typology. Studia Typologica 6. Berlin, Akademie. https://ai2-s2pdfs.s3.amazonaws.com/9bcf/206e40b9d852a9694f4b75bcddea002d1059.pdf [11.01.2014].

Fanselow, Gisbert (2002): “Quirky 'Subjects' and other Specifiers”. In: Kaufmann, Ingrid/Stiebels, Barbara (eds.): More than words. A Festschrift for Dieter Wunderlich. Berlin, Akademie: 227-250. (= Studia Grammatica 53).

Firbas, Jan (1964): “On Defining the Theme in Functional Sentence Analysis". Travaux linguistiques de Prag 1: 267-280.

Frey, Werner (2006): "How to Get an Object-es into the German Prefield". In: Brandt, Patricks/Fuss, Eric (eds.): Form, Structure, and Grammar - A Festschrift Presented to Günther Grewendorf on Occasion of His 60th Birthday. Berlin, Akademie: 159-185. 
Geilfuß, Jochen (1991): „Jiddisch als SOV-Sprache“. Working Papers of Sonderforschungsbereich 340. Universities of Stuttgart and Tübingen: 3-17.

Geller, Ewa (2004): „Jiddisch im Spannungsfeld zwischen Germanistik und Slawistik“. Convivium. Germanistisches Jahrbuch Polen. Polendiskurse:171-197.

Grohmann, Kleanthes (ed.) (2009): Explorations of Phase Theory: Features and Arguments. Berlin: de Gruyter.

Haider, Hubert ( $\left.{ }^{2} 1992\right)$ : „Über sein oder nicht sein: Zur Grammatik des Pronomens sich“. In: Abraham, Werner (ed.): Erklärende Syntax des Deutschen. Tübingen, Narr: 223-254.

Haider, Hubert/Szucsich, Luka (2012): Scrambling and V-Positioning in Slavic Languages Exceptionally VO or Regular T3? www.uni-salzburg.at/fileadmin/oracle_file_imports /2145553.PDF [16.06.2015].

Hentschel, Elke (2003) "Es war einmal ein Subjekt". Linguistik online 13, 1/03. www.linguistik-online.de/13_01/hentschel.html [15.01.2015].

Jacobs, Neil (2005): Yiddish. A Linguistic Introduction. Cambridge: Cambridge University Press.

Kühnert, Henrike/Wagner, Ester-Miriam (2014): "Changes in the position of the finite verb in older Yiddish”. In: Aptroot, Marion/Hansen, Björn (eds.): Yiddish Language Structures. Berlin/Boston, de Gruyter: 125-142.

Koster, Jan (1978): Locality Principles in Syntax. Dordrecht: Foris Publications.

Levin, Beth/Rappaport Hovav, Malka (1995): Unaccusativity. Cambridge: MIT.

Mark, Yudl (1978): Gramatik fun der yidisher klal-shprakh. New York: Alveltlekher Yidisher Kulturkongres.

Mohr, Sabine (2005): Clausal Architecture and Subjects Positions: Impersonal Constructions in the Germanic Languages. Amsterdam: Benjamins.

Perlmutter, David (1978): "Impersonal Passives and the Unaccusative Hypothesis". Proceedings of the $4^{\text {th }}$ Annual Meeting of the Berkeley Linguistics Society: 157-190.

Pollock, Jean-Yves (1989): "Verb Movement, Universal Grammar, and the Structure of IP". Linguistic Inquiry 20/3: 365-424.

Prince, Ellen (1989): "Yiddish wh-Clauses, Subject-Postponing, and Topicalization". In: Powers, Joyce/Jong de, Ken (eds.): ESCOL 88. Columbus, Ohio State University: 403415.

Prince, Ellen (1993): "On the discourse functions of syntactic form in Yiddish: expletive es and subjectpostponing”. In: Goldberg, David/Herzog, Marvin/Kirshenblatt-Gimblett, Barbara/Miron, Dan (eds.): The field of Yiddish. Studies in Yiddish Language, Folklore, and Literature, $5^{\text {th }}$ collection. Evanston, Northwestern University Press and YIVO Institute for Jewish Research: 59-86.

Reinhart, Tanya (1981): "Pragmatics and Linguistics: an Analysis of Sentence Topics". Philosophica 27: 53-94.

Reinhart, Tanya (1996): "Syntactic Effects of Lexical Operations: Reflexives and Unaccusatives". OTS Working Papers in Linguistics. University of Utrecht.

Reinhart, Tanya/Siloni, Tal (2005): "The Lexikon-Syntax Parameter: Reflexivization and Other Arity Operations". Linguistic Inquiry 36/3: 389-436.

Reis, Marga (1982): „Reflexivierung im Deutschen“. In: Faucher, Eugène (ed.)(1981): Actes du Colloque du Centre de Recherches Germaniques de l'Université de Nancy et Journée 
Annuelle des Linguistes de l'Association des Germanistes de l'Enseignement Supérieur 12. Décembre, Nancy. 1-40.

Rizzi, Luigi (1997): "The Fine Structure of the Left Periphery". In: Haegeman, Liliane (ed.): Elements of Grammar; Handbook in Generative Syntax. Dordrecht, Kluwer: 281-338.

Rizzi, Luigi (ed.) (2004): The Structure of CP and IP. The Cartography of Syntactic Structures. Vol. 2. New York: Oxford University Press.

Rizzi, Luigi (2005): “On some Properties of Subjects and Topics”. In: Brugè, Laura et al. (eds.): Contributions to the $30^{\text {th }}$ Incontro di Grammatica Generativa, Venice, February 26-28, 2004. Venezia, Università Ca' Foscari: 203-224.

Reis, Marga (1999): "On Sentence Types in German: an Enquiry into the Relationship between Grammar and Pragmatics". Interdisciplinary Journal for Germanic Linguistics and Semiotic Analysis 4/2: 195-236.

Sadock, Jerrold (1998): “A Vestige of Verb Final Syntax in Yiddish". Monatshefte 90/2: 220 226.

Santorini, Beatrice (1992): "Variation and Change in Yiddish Subordinate Clause Word Order". Natural Language and Linguistic Theory 10: 595-640.

Santorini, Beatrice (1993): "The Rate of Phrase Structure Change in the History of Yiddish". Language Variation and Change 5: 257-283.

Sgall, Petr (1972): “Topic, Focus and the Ordering of Elements of Semantic Representation". Philologica Pragensia 15: 1-14.

Shlonsky, Ur (2010): “The Cartographic Enterprise in Syntax". Language and Linguistics Compass 4/6: 417-429.

Sigurdsson, Halldór (1996): "Icelandic Finite Verb Agreement". Working Papers in Scandinavian Syntax 57: 1-46.

Vikner, Sten (2013) "Verb Particles in Germanic SVO- and SOV-Languages: Yiddish Compared to Danish and German". SyntaxLab. University of Cambridge. www.hum.au.dk/engelsk/engsv/handouts/vikner-ho-2013-Cambridge-particles.pdf [09.03.2014].

Wallenberg, Joel (2013): "Scrambling, LF, and Phrase Structure Change in Yiddish". Lingua 133: 289-318. 